\title{
Radically different maxicircle classes within the same kinetoplast: an artefact or a novel feature of the kinetoplast genome? Pavel N Flegontov ${ }^{\dagger}$ and Alexander A Kolesnikov*†
}

\author{
Address: Department of Molecular Biology, Lomonosov Moscow State University, Vorobjevy Gory 1, Build. 12, 119992 Moscow, Russia \\ Email: Pavel N Flegontov - flegontov@list.ru; Alexander A Kolesnikov* - sasha@protein.bio.msu.ru \\ * Corresponding author †Equal contributors
}

Published: 18 September 2006

Kinetoplastid Biology and Disease 2006, 5:5 doi:10.1 186/1475-9292-5-5

This article is available from: http://www.kinetoplastids.com/content/5/l/5

(C) 2006 Flegontov and Kolesnikov; licensee BioMed Central Ltd.

This is an Open Access article distributed under the terms of the Creative Commons Attribution License (http://creativecommons.org/licenses/by/2.0), which permits unrestricted use, distribution, and reproduction in any medium, provided the original work is properly cited.
Received: 14 July 2006

Accepted: 18 September 2006

\begin{abstract}
We discuss here some results which suggest that radically different maxicircle classes coexist within the same kinetoplast. These data, although tentative and incomplete, may provide a new outlook on the kinetoplast genome structure and expression.
\end{abstract}

\section{Report}

The kinetoplast genome is composed of a large heterogeneous population of minicircles and a small number of maxicircles. The maxicircles contain a conserved coding region of 16-17 kb and a non-coding "divergent" region which is highly variable in different strains and species [111]. It is believed that the population of maxicircles within a single mitochondrion is homogeneous.

The first results contradicting this widely accepted assumption were obtained by Lee et al. in the early 90-es [12-16]. Drastic changes of kinetoplast DNA were observed in strains of Leishmania mexicana amazonensis selected in vitro for arsenite or tunicamycin resistance, and these changes were accompanied by amplification of extrachromosomal DNA in the nucleus [12]. It was demonstrated that a new maxicircle DNA variant appeared in all drug-resistant strains along with the extrachromosomal DNA amplification. This variant was characterized by an altered pattern of restriction sites $[12,13]$, it had a radically different sequence of the cytochrome $b$ gene [13] and also displayed extensive DNA sequence rearrangements in the divergent region [16]. Nevertheless, the mutant cells demonstrated a normal growth pattern and apparently retained functioning mitochondria [13].
Moreover, alterations of the minicircle kinetoplast DNA component ("minicircle dominance switch") were also observed: the predominant wild-type minicircle class was replaced by other minicircle classes in the drug-resistant cells [12-15]. It was hypothesized that the switch of minicircle dominance might alter editing pathways and help to generate functional proteins from the altered maxicircle sequence (for example, of the cytochrome $b$ gene) [13]. Remarkably, very similar maxicircle variants appeared in different cloned cell lines independently selected for arsenite or tunicamycin resistance $[13,16]$. This fact lead the authors to suppose that there was more than one maxicircle type in the kinetoplast DNA network in all studied cell lines, and a minor maxicircle variant became prevalent after the selection for drug resistance [13]. Thus, the switch of maxicircle dominance might accompany the switch of minicircle dominance. This conclusion remains controversial because, rather unexpectedly, minor maxicircle classes were not detected directly by PCR or hybridization in the wild-type or drug-resistant strains and because the results of Lee et al. were never repeated by another group. However, no alternative mechanisms underlying the observed drastic changes of the kinetoplast DNA were proposed. 
The new results recently obtained by us [9] on a related species, Leishmania major, shed an additional light on the potential phenomenon encountered by Lee et al. with $L$. $m$. amazonensis. We observed that the promastigote-amastigote differentiation in vivo is accompanied by an alteration of the maxicircle divergent region sequence [9]. The DR structure proved to be drastically different at the promastigote and amastigote stages. Large-scale sequence rearrangements and also multiple small-scale insertions/ deletions and single nucleotide substitutions were observed. Most remarkably, several strains of independent origin demonstrated an identical rearrangement pattern suggesting that the hypothetical "switch of maxicircle dominance" might take place during the differentiation. However, our results remain tentative, and this work needs to be repeated in vitro using clonal cell lines.

It is not known but possible that maxicircle genome activity differs between the amastigote and promastigote stages. We hypothesized that the structure of the divergent and/or the coding region in the prevailing maxicircle class may determine the pattern of genome activity. Thus, a novel regulatory mechanism may act in the kinetoplast of Leishmania: a specialized genome for each life cycle stage. This mechanism remains purely hypothetical, but because of its novelty and importance it deserves a thorough experimental verification.

The discussed results suggest that: 1) maxicircles may be intrinsically heterogeneous (at least in some Leishmania species); 2) different maxicircle classes may coexist within a single kinetoplast (since the strains of $L$. m. amazonensis used by Lee et al. were clonal); 3 ) maxicircle classes may have different divergent region structure and even different sequences of some genes; 4 ) a switch of the predominant maxicircle class accompanied by shifts in the abundance of minicircle classes might lead to alteration of editing patterns; 5) the maxicircle and minicircle dynamics might represent a novel mechanism regulating the kinetoplast gene expression. These hypotheses provide a new outlook on the kinetoplast genome expression and, therefore, deserve rigorous testing.

\section{Conclusion}

We hypothesize that a switch of the predominant maxicircle class may take place during the promastigote-amastigote differentiation and under drug pressure. The data discussed here, although ambiguous and incomplete, suggest that a novel regulatory mechanism may operate in the kinetoplasts. Subsequent studies will either confirm the coexistence of radically different maxicircle classes within the same kinetoplast or provide an alternative explanation for the observed kinetoplast DNA alterations.

\section{Competing interests}

The author(s) declare that they have no competing interests.

\section{References}

I. Borst P, Fase-Fowler F, Hoeijmakers JHJ, Frasch ACC: Variations in maxi-circle and mini-circle sequences in kinetoplast DNAs from different Trypanosoma brucei strains. Biochim Biophys Acta 1980, 610:197-210.

2. Borst P, Weijers P, Brakenhoff B: Analysis by electron microscopy of the variable segment in the maxi-circle of kinetoplast DNA from Trypanosoma brucei. Biochim Biophys Acta 1982, 699:272-280

3. Muhich ML, Simpson L, Simpson AM: Comparison of maxicircle DNAs of Leishmania tarentolae and Trypanosoma brucei. Proc Natl Acad Sci USA 1983, 80:4060-4064.

4. Maslov DA, Kolesnikov AA, Zaitseva GN: Conservative and divergent base sequence regions in the maxicircle kinetoplast DNA of several trypanosomatid flagellates. Mol Biochem Parasitol 1984, I 2:35I-364.

5. Muhich ML, Neckelmann N, Simpson L: The divergent region of Leishmania tarentolae kinetoplast maxicircle DNA contains a diverse set of repetitive sequences. Nucleic Acids Res 1985, I3:324I-3260.

6. Simpson L, Neckelmann N, de la Cruz VF, Simpson AM, Feagin JE, Jasmer DP, Stuart K: Comparison of the maxicircle (mitochondrial) genomes of Leishmania tarentolae and Trypanosoma brucei at the level of nucleotide sequence. J Biol Chem 1987, 262:6182-6196.

7. Sloof $P$, de Haan A, Eier W, van lersel M, Boel E, van Steeg H, Benne $R$ : The nucleotide sequence of the variable region in Trypanosoma brucei completes the sequence analysis of the maxicircle component of mitochondrial kinetoplast DNA. Mol Biochem Parasitol 1992, 56:289-299.

8. Myler PJ, Glick D, Feagin JE, Morales TH, Stuart KD: Structural organization of maxicircle variable region of Trypanosoma brucei : identification of potential replication origins and topoisomerase II binding sites. Nucleic Acids Res 1993, 21:687-694.

9. Flegontov PN, Strelkova MV, Kolesnikov AA: The Leishmania major maxicircle divergent region is variable in different isolates and cell types. Mol Biochem Parasitol 2006, I46:173-179.

10. Westenberger S], Cerqueira GC, El-Sayed NM, Zingales B, Campbell DA, Sturm NR: Trypanosoma cruzi mitochondrial maxicircles display species- and strain-specific variation and possess a conserved element in the non-coding region. BMC Genomics 2006, 7:60.

II. Flegontov PN, Guo Q, Ren L, Strelkova MV, Kolesnikov AA: Conserved repeats in the kinetoplast maxicircle divergent region of Leishmania sp. and Leptomonas seymouri. Mol Genet Genomics 2006 in press.

12. Lee SY, Lee ST, Chang KP: Transkinetoplastidy: A novel phenomenon involving bulk alterations of mitochondrial kinetoplast DNA of a trypanosomatid protozoan. J Protozool 1992, 39:190-196.

13. Lee ST, Tarn C, Wang CY: Characterization of sequence changes in kinetoplast DNA maxicircles of drug-resistant Leishmania. Mol Biochem Parasitol 1992, 56:197-208.

14. Lee ST, Tarn C, Chang KP: Characterization of the switch of kinetoplast DNA minicircle dominance during development and reversion of drug resistance in Leishmania. Mol Biochem Parasitol 1993, 58:187-204.

15. Lee ST, Liu HY, Lee SP, Tarn C: Selection for arsenite resistance causes reversible changes in minicircle composition and kinetoplast organization in Leishmania mexicana. Mol Cell Biol 1994, 14:587-596.

16. Lee ST, Liu HY, Chu T, Lin SY: Specific A+T-rich repetitive DNA sequences in maxicircles from wildtype Leishmania mexicana amazonensis and variants with DNA amplification. Exp Parasitol 1994, 79:29-40. 